\title{
CAPACIDADE DE REPLICAÇÃO DA CEPA Y Trypanosoma cruzi EM DIFERENTES ESPÉCIES DE TRIATOMÍNEOS
}

\author{
Ionizete Garcia da Silva ${ }^{*}$, Leila Gonçalves Pereira dos Santos ${ }^{* *}$, Rejane Nakano ${ }^{* *} e$ \\ Renato Calixto Badauy"
}

RESUMO

A replicação da cepa $Y$ de Trypanosoma cruzi, em diferentes espécies de triatomíneos, foi estudada através do xenodiagnóstico, aplicado em camundongos com a infecção aguda. Utilizaram-se 20 camundongos infectados e oito espécies de triatomíneos. Cada triatomíneo foi examinado individualmente, pelo método das dejeções espontâneas. A capacidade, das diferentes espécies de triatomíneos, de replicar o T.cruzi, utilizando como parâmetro de avaliação o número de tripanosomas excretados encontra-se em ordem decrescente: Dipetalogaster maximus, Panstrongylus herreri, Triatoma rubrovaria, T. tibiamaculata, T. matogrossensis, T.protracta, T.sordida, Rhodnius prolixus. Os experimentos foram realizados numa câmara biológica, a $28 \pm 1{ }^{\circ} \mathrm{C}$, umidade relativa de $70 \pm 5 \%$ e fotoperíodo de 12 horas.

UNITERMOS:Replicação. Triatomíneos. Trypanosoma cruzi. Tripanosomíase americana.

\section{INTRODUÇÃO}

O xenodiagnóstico tem sido utilizado tanto para diagnosticar a tripanosomíase americana, no homem e em outros mamíferos, quanto para demonstrar a parasitemia circulante em chagásicos que se encontram em tratamento com drogas tripanosomicidas. Isto tem estimulado várias investigações, com a finalidade de se conhecer a capacidade de diferentes espécies de triatomíneos de replicar o Trypanosoma cruzi. Dentre essas, pode-se citar os estudos das relações biológicas entre triatomíneo e tripanosoma, na busca do modelo experimental.

\footnotetext{
Prof. do Depto. de Parasitologia - IPTSP/UFG.

- Bolsita de iniciação científica do CNPq

Apoio financeiro: CONSITEG, SENESU, FUNAPE.

Recebido para publicação em 14/11/94
} 
SILVA,I.G.; SANTOS,L.G.P.; NAKANO, R.; BADAUY, R.C. Capacidade de Replicação da Cepa $Y$ Trypanosoma cruzi em Diferentes Espécies de Triatomíneos. Rev.Pat.Trop.23 ( 2 ):197204,jul./dez. 11994

Nleste trabalho, estudou-se a capacidade de replicação de espécies tipicamente silvestres, Dipetalogaster maximus e Triatoma tibiamaculata; silvestre que invade domicílio sem colonizá-lo, T.protracta; em processo de adaptação ao domicílio humano, Panstrongylus herreri, T.matogrossensis, T. rubrovaria, e que colonizam tanto ecótopos naturais como artificiais, Rhodnius prolixus e T.sordida, em relação cepa $Y$ de T.cruzi, na busca da espécie de triatomíneo mais adequada para replicar este flagelado, com a finalidade de usá-la em rotina no xenodiagnóstico. A utilização dessa espécies, teve como objetivo estudar a relação triatomíneo-tripanosoma, associada aos hábitos nos diferentes ecótopos com a replicação do tripanosoma, aproveitando a disponibilidade de triatomíneo no laboratório.

\section{MATERIAL E MÉTODOS}

\section{Criação dos triatomíneos}

Os triatomíneos foram criados numa câmara biológica à temperatura de $28 \pm$ $1{ }^{\circ} \mathrm{C}$, umidede relativa de $70 \pm 5 \%$ e fotoperíodo de $12 \mathrm{~h}^{12}$.Utilizaram-se para criação, frascos plásticos cilíndricos, azuis, semitransparentes, resultantes do aproveitamento de garrafas d'ćgua, medindo $9,0 \mathrm{~cm}$ de diâmetro por $14,5 \mathrm{~cm}$ de altura ${ }^{12}$.Todas as espécies de triatomneos, com exceção de D.maximus, foram alimentadas em Gallus gallus domesticus(galinha) 1216

\section{Inoculaçãc de camundongos e xenodiagnóstico}

Fcram inoculados, por via intraperitoneal, 20 camundongos machos, albinos, isogênicos, da linhagem $\mathrm{A} / \mathrm{Sn}$, com cerca de 5 semanas de vida, com $1,0 \times 10^{5}$ formas sangÜíneas da cepa $Y$ de T.cruzi. No $8^{\circ}$ dia após a inoculação, seccionou-se a extremidad da cauda do camundongo, colheu-se $5 \mathrm{ml}$ de sangue com um micropipet:dor automático e contou-se $0 \mathrm{n}^{0}$ de tripanosomas circulantes ${ }^{1} 14$ Imediatamente após a contagem de tripanosomas, realizou-se o xenodiagnóstico; para isto, cada camundongo foi imobilizado numa tela de náilon ${ }^{14}$, e afixado aos tubos com um triatomneo de cada espécie, através de elásticos, possibilitando um permanente contato con a fonte de alimentação.

A: leituras do xenodiagnóstico foram realizadas aos 30,60,90,120 e 150 dias, após : aplicação do exame, pela técnica das dejeções espontâneas ${ }^{13}$. Esta técnica permite exaninar separadamente, tanto as fezes quanto a urina, para isto é fundamental que o triatoníneo atinja a repleção total ${ }^{16}$. Trypanosoma cruzi em Diferentes Espécies de Triatomíneos. Rev.Pat.Trop.23 ( 2 ):197. 204,jul./dez.1994

Testaram-se 8 espécies, com 20 triatomíneos cada, em jejum absoluto, sendo que usou-se D.maximus no $1^{\circ}$ estádio, dez dias após eclosão das ninfas; P.herreri, T.matogrossensis, T.rubrovaria, T.sordida e T.tibiamaculata, 12 dias após a ecdise do $2^{\circ}$ estádio e, R.prolixus e T.protracta, 15 dias após ecdise do $3^{\circ}$ estádio.Estes períodos de jejum foram constatados, numa experiência piloto, como os mais favoráveis para que o triatomíneo atinja a repleção total.

A suscetibilidade foi estimada através de dois parâmetros: número de triatomíneos infectados e número de tripanosomas excretados, pela leitura individualizada de cada triatomíneo.

\section{RESULTADOS}

Tabela 1 - Peso médio e quantidade média de sangue ingerida por triatomíneos, alimentados $\mathrm{em}$ camundogos infectados pelo Trypanosoma cruzi, com a infecção aguda.

\begin{tabular}{lcc}
\hline ESPÉCIES & Peso(mg) & Sangue Ingerido(ml) \\
\hline Dipetalogaster maximus & $07 \pm 0,35$ & $63 \pm 6.07$ \\
Panstrongylus herreri & $11 \pm 3,25$ & $41 \pm 5,11$ \\
Triatoma rubrovaria & $09 \pm 0,35$ & $40 \pm 5,21$ \\
Triatoma tibiamaculata & $06 \pm 0,49$ & $30 \pm 5,62$ \\
Triatoma matogrossensis & $10 \pm 0,21$ & $45 \pm 6,51$ \\
Rhodnius prolixus & $06 \pm 0,53$ & $64 \pm 6,58$ \\
Triatoma sordida & $09 \pm 0,43$ & $45 \pm 5,25$ \\
Triatoma protracta & $06 \pm 0,37$ & $39 \pm 1,31$ \\
\hline
\end{tabular}

Tabela 2. Frequência (\%) de triatomíneos infectados pelo Trypanosoma cruzi, avaliada pelo xenodiagnóstico,aplicado em camundongos com a infecção aguda.

\begin{tabular}{lccccc}
\hline & \multicolumn{5}{c}{$\%$ de triatomíneos infectados/leitura-dia } \\
\cline { 2 - 6 } Espécies & 30 & 60 & 90 & 120 & 150 \\
Dipetalogaster maximus & 100 & 100 & 100 & 100 & 100 \\
Panstrongylus herreri & 100 & 100 & 100 & 100 & 100
\end{tabular}


SILVA,I.G.; SANTOS,L.G.P.; NAKANO, R.; BADAUY, R.C. Capacidade de Replicação da Cepa $Y$ Trypates Espécies de Triatomíneos. Rev.Pat.Trop.23 ( 2 ):197204.jul./dez.1994

\begin{tabular}{lrrrrr} 
Triatoma rubrovaria & 100 & 100 & 100 & 100 & 100 \\
Triatoma tibiamaculata & 100 & 100 & 100 & 100 & 100 \\
Triatoma matogrossensis & 100 & 100 & 100 & 100 & 100 \\
Rhodnius prolixus & 100 & 100 & 100 & 100 & 100 \\
Triatoma sordida & 40 & 75 & 75 & 85 & 90 \\
Triatoma protracta & 20 & 30 & 40 & 45 & 55 \\
\hline
\end{tabular}

Tabela 3. Número médio de Trypanosoma cruzi ingeridos e excretados por triatomíneos, estimado através do xenodiagnóstico, usando a técnica das dejeçð̃es espontâneas.

\begin{tabular}{|c|c|c|c|c|c|c|}
\hline \multirow{3}{*}{ Espécies } & \multicolumn{6}{|c|}{$\mathrm{N}^{0}$ médio de Trypanosoma cruzi em $5 \mathrm{ml}$} \\
\hline & \multirow{2}{*}{$\begin{array}{l}\text { ingeridos } \\
\text { (sangue) }\end{array}$} & \multicolumn{5}{|c|}{ excretados(fezes)/leituras em $5 \mathrm{ml}$} \\
\hline & & 30 & 60 & 90 & 120 & 150 \\
\hline D. maxim's & $4,1 \times 10^{5}$ & 1.544 & 7.097 & 13.093 & 4.177 & 6.926 \\
\hline P. herreri & $2,8 \times 10^{5}$ & 52 & 3.647 & 5.109 & 2.614 & 4.844 \\
\hline T. rubrovaria & $2,1 \times 10^{5}$ & 11 & 1.498 & 4.746 & 6.091 & 6.722 \\
\hline T. tibiamcculata & $6,9 \times 10^{5}$ & 1.949 & 619 & 1.741 & 1.201 & 1.565 \\
\hline T. matogmossensis & $3,2 \times 10^{5}$ & 326 & 415 & 2.486 & 237 & 1.528 \\
\hline T. protracta & $2,2 \times 10^{5}$ & 15 & 03 & 7.523 & 235 & 228 \\
\hline T. sordide & $2,9 \times 10^{5}$ & 36 & 990 & 28 & 20 & 961 \\
\hline R. prolixus & $4,3 \times 10^{5}$ & 33 & 22 & 217 & 370 & 17 \\
\hline
\end{tabular}

D.= Dipealogaster,$P .=$ Panstrongylus, $T .=$ Triatoma

1 Tabela 3 mostra a eliminação de tripanosomas pelos triatomíneos, que oscilou d uma leitura a outra, assim detectava-se numa leitura grande quantidade de tripanosonas e, às vezes, isso não se repetia na seguinte ou não eliminava nenhum flagelado pelo exame individualizado do trkatomíneo. Nos 5 meses estudados, após a infecção dos triatomíneos, verificou-se que a eliminação de T.cruzi foi significatvamente maior $(p<0,05)$ nos $2^{\circ}$ e $3^{\circ}$ meses, em todas as espécies. Verificou-se que a qantidade de sangue ingerida não foi fator determinante do número de
SILVA,I.G.; SANTOS,L.G.P.; NAKANO, R.; BADAUY, R.C. Capacidade de Replicaço da Cep Y Trypanosoma cruzi em Diferentes Espécies de Triatomíneos. Rev.Pat.Trop.23 ( 2 ):197.
204,jul./dez.1994

tripanosomas excretados pelos triatomíneos, e que apenas o parâmetro, número de 14 tripanosomas excretados, determinou um gradiente da capacidade de replicação e se encontra na seguinte ordem:D.maximus, P.herreri, T.rubrovaria, T. tibiamaculata, T. matogrossensis, T.protracta, T. sordida, R.prolixus, pela análise de variância, ao nível de $5 \%$.

\section{DISCUSSÃO}

A relação biológica, triatomíneo-T.cruzi tem sido estudada por vários autores. Um grupo mostrou que a suscetibilidade é maior em triatomíneos da mesma regiåo geográfica das cepas do tripanosoma ${ }^{34511}$. No entanto, os resultados de outros grupos ${ }^{15}$ ${ }^{20}$ e deste trabalho, com D. maximus, P.herreri e T.rubrovaria, são discordantes e inversos aos anteriores.

Da relação biológica triatomíneo-tripanosoma, surgiram vários trabalhos, o primeiro deles, mostrou que os índices de infecção pelo T.cruzi crescia com os estádios ninfais dos triatomíneos ${ }^{19}$. Desta época para cá, provavelmente tenha surgido a hipótese da importância da quantidade de sangue ingerida e o número de flagelados excretados.Isto parece ter induzido, àqueles que trabalham com o xenodiagnóstico, a utilizarem triatomíneos em estádios mais avançados $\left(4^{\circ}\right.$ e $\left.5^{\circ}\right)$. Posteriormente, aparece um trabalho ${ }^{8}$ com resultados inversos, e um outro ${ }^{10}$ mostrando que não havia uniformidade quanto aos indices de infecção pelo T.cruzi quando a quantidade de tripanosomas era alta.Tem sido demonstrado em vários artigos ${ }^{671417}$ que a quantidade de sangue ingerida não é fator determinante na infecção do triatomíneo. Este fato é concordante com os resultados deste trabalho.

Outro aspecto importante da suscetibilidade de triatomíneos ao T.cruzi, relaciona-se com as formas do tripomastigota, largas e finas, de diversas cepas, e que, triatomíneos se infectavam melhor com as cepas nas quais predominavam as formas tripomastigotas largas ${ }^{2}$. Neste trabalho, utilizou-se a $Y$ de $T$.cruzi, que se caracteriza pelo predomínio de formas finas, pelo desafio que ela apresentava em desenvolver menor infecção nos triatomíneos, pela hipótese de melhor selecionar a suscetibilidade dos triatomíneos, bem como, pela facilidade de infectar animais de laboratório.

Outro estudo relevante para determinação da suscetibilidade, refere-se às técnicas de leituras coproscópicas. O estudo de duas técnicas de leituras, a partir do xenodiagnóstico aplicado em 206 pacientes chagásicos crônicos, mostrou que o método das dejeções espontâneas (m.d.e.) era mais eficiente do que o método da compressão abdominal (m.c.a.) para detectar o T.cruzi, com isso conseguiu melhorar a sensibilidade do exame ${ }^{13}$. Utilizando o m.d.e., neste trabalho, verificou-se que as espécies D.maximus, P.herreri e T.rubrovaria, foram os melhores meios replicadores do T.cruzi. A fonte de infecção pode ser mais uma variável no estudo da suscetibilidade. As espécies domiciliadas, T.infestans e T.brasiliensis, eram mais suscetíveis ao T.cruzi 
SILVA,I.G.; SANTOS,L.G.P.; NAKANO, R.; BADAUY, R.C. Capacidade de Replicação da Cepa $Y$ Trypanosoma cruzi em Diferentes Espécies de Triatomíneos. Rev.Pat.Trop.23 ( 2 ):197204,jul./dez.1994

da área endêmica chagásica de São Felipe - Bahia, usando como fonte de infecção o cão e o tatu ${ }^{18}$.Usando o cobaio $^{8}$ como fonte de infecção para os triatomíneos, as espécies silvestres se infectavam melhor do que as domiciliadas, sendo $P$. megistus a mais suscetível ao $T$. cruzl $^{8}$. Neste trabalho, utilizaram-se camundongos isogênicos $\mathrm{A} / \mathrm{Sn}$ como fonte de infecção, e constatou-se que a espécie tipicamente silvestre, $D$. maximus, foi a mais suscetível, seguida de outras silvestres que se encontram em processo de domiciliação, P.herreri e T.rubrovaria. A espécie que apresenta o maior grau de domiciliação, R.prolixus, entre as estudadas, teve o pior desempenho na replicação do $T$. cruzi.

Existem diversos fatores relacionados com a suscetibilidade de triatomíneos ao T.cruzi, porém neste trabalho, estudaram-se as variáveis: número de triatomíneos infectados, sangue ingerido, tripanosomas ingeridos e excretados, evidenciando D.maximus como o melhor replicador.Isto é concordante com outros estudos recentes ${ }^{15}$

\section{SUMMARY}

Difference in capacity of replication to $Y$ strain of Trypanosoma cruzi among 8 species of triatomines.

Capacity of replication of different triatomine species was investigated by applying xenodiagnosis in mice during acute Trypanosoma cruzi infection ("Y" strain). Twenty insect bugs for each one of the studied species were applied on infected mice. Each bug was examined individually.

The species capacity of replication in relation with the number of the excreted trypanosomes was : Dipetalogaster maximus, Panstrongylus herreri, Triatoma rubrovaria, T.tibiamaculata, T.matogrossensis, T. protracta, T. sordida and Rhodnius prolixus.

The experiments were performed at $28 \pm 1^{\circ} \mathrm{C}, 70 \pm 5 \%$ of relative humidity and photoperiod of $12 \mathrm{~h}$.

KEYWORDS: Repl:cation. Triatomines.Trypanosoma cruzi. Chagas' disease.

\section{REFERÊNCIAS BIBLIOGRÁFICAS}

1. BRENER,Z. Coniribuição ao estudo da terapêutica experimental da doença de Chagas. Tese de Docência-Livre. Faculdade de Odontologia e Farmáci. de Minas Gerais, Belo Horizonte, 1961.
SILVA,I.G.; SANTOS,L.G.P.; NAKANO, R.; BADAUY, R.C. Capacidade de Replicaçđo da $\frac{203}{2}$ Trypanosoma cruzi em Diferentes Espécies de Triatominos. Rev.l 204,jul./dez.1994

2. BRENER,Z. Life cycle of Trypanosoma cruzi. Rev.Inst.Med. Trop. S.Paulo, 13: 171-178, 1971.

3. CERISOLA,J.A.; RHOWEDDER,R.W. \& PRADO, C.E. Rendimento del xenodiagnóstico en la infeccion cronica humana utilizando ninfas de diferentes especies de triatomineos.Bol.Chil. Parasit., 26:57-58, 1971.

4. DIAS, E. Xenodiagnósticos seriados em cães infectados com amostras venezuelanas de "Schizotrypanum cruzi". Brasil Médico, 54:859-861, 1940. Replicação da Cepa $Y$ Trypanosoma cruzi em Diferentes Espécies de Triatomíneos. Rev.Pat.Trop.23(2):183-188,jul./dez.1994

5. LITTLE,J.W.; TAY, J. \& BIAGGI, F. A Study on the suscetibility of triatominae bugs to some Mexican strains of Trypanosoma cruzi.J.Med.Ent., 3:252-255, 1966.

6. MELLO,D.A \& CHIARINI,C. Suscetibilidade dos diferentes estádios evolutivos de Triatoma sordida (Stal,1859) e de Rhodnius neglectus lent, 1954, à infecção pelo Trypanosoma cruzi. Rev.Bras.Biol., 40:327-334, 1980.

7. MILES,M.A.; PATTERSON,J.W.; MARSDEN, P.D. \& MINTER, M.D. A comparison of Rhodnius prolixus, Triatoma infestans and Panstrongylus megistus in the xenodiagnosis of a chronic Trypanosoma (Schizotrypanum) cruzi infection in a Rhesus monkey (Macaca mulata).Trans.Roy.Soc.Trop.Med.Hyg., 69:377- 382,1975.

8. NEAL, R.A. \& MILES,R.A. The numbers of trypomastigotes of Trypanosoma cruzi required to infect Rhodnius prolixus. Rev.Inst.Med.Trop., S.Paulo, 19:177 $181,1977$.

9. PERLOWAGORA-SZUMLEWICZ, A.; MÜLLER, C.A. \& MOREIRA, C.J.C Studies in search of a suitable experimental insect model for xenodiagnosis of hosts with chagas'disease. 3 - on the interaction of vector species and parasite strain in the reaction of bugs to infection by Trypanosoma cruzi. Rev.Saúde Púb., 22:390-400, 1988.

10. PHILLIPS,N.R. \& BERTRAN,D.S. Laboratory studies of Trypanosoma cruzi infections in:Rhodnius prolixus - larvae and adults; in: Triatoma infestans, $T$. protracta and T. maculata - adults.J.Med.Ent., 4:168-174, 1967. 
SILVA,I.G.; SANTOS,L.G.P.; NAKANO, R.; BADAUY, R.C. Capacidade de Replicação da Cepa $Y$ Trypanosoma cruzi em Diferentes Espécies de Triatomíneos. Rev.Pat.Trop.23 ( 2 ):197204.jul./dez.1994

11. RYCKMAN,R.E. Epizootiology of Trypanosoma cruzi in South western North America.V.Host parasite specificity between Trypanosoma and Triatominae (Kinetoplastide: Tripanosomatidae) (Hemiptera:Triatominae). J.Med.Ent., 2:96-99,1965.

12. SILVA,I.G.da Influência da temperatura na biologia de triatomíneos.I.Triatoma rubrovaria (Blanchard,1843) (Hemiptera, Reduviidae).Rev.Goiana Med., 31:137, 1985.

13. SILVA, I.G.da Nova técnica para a leitura do xenodiagnóstico. Rev.Goiana Med., 36:35-39, 1990.

14. SILVA, I. G.da \& FERREIRA,I.R. Influência da fonte sanguínea na multiplicação da cepa Y de Trypanosoma cruzi em Triatoma infestans (Klug,1834) e Rhodnius neglectus Lent,1954. Rev. Goiana Med., 36:41-48, 1990.

15. SILVA,I.G.da; OSTERMAYER,A.L.; SILVA,H.H.G.da Importância do método de obtenção das dejeções dos triatomíneos na avaliação da suscetibilidade triatomínica ao Trypanosoma cruzi.Rev.Soc. Bras.Med.Trop., 26:19-24, 1993.

16. SILVA,I.G. \& SILVA, H.H.G.da Estudo comparado do desenvolvimento de triatomíneos a duas temperaturas. Mem.Inst.Oswaldo Cruz, 84:499-500, 1989.

17. SILVA,I.G. \& SILVA,H.H.G. Suscetibilidade de 11 espécies de triatomíneos (Hemiptera, Reduviidae) à cepa Y de Trypanosoma cruzi (Kinetoplastida, Tripanosomatidae).Rev.Bras.Ent.37:459-463, 1993.

18. SHERLOCK, I. A. \& ALMEIDA,S.A. Diferença de suscetibilidade à infecção com T.cruzi entre espécies de triatomíneos alimentados em cão, tatu e camundongo infectados. Rev. Soc.Bras.Med.Trop., 7:87-98, 1973.

19. TORRES, M. Alguns fatos que interessam a epidemiologia da moléstia de Chagas.Mem.Inst.Oswaldo Cruz, 7:120- 136, 1915.

20. ZELEDÓN,R. Vieto PL.Susceptibilidad de varias espécies de triatomínos a uma cepa costarricence de Schizotrypanum cruzi.Rev.Biol.Trop., 5:195-199, 1957. 